\title{
Dysplasia of the Upper Aerodigestive Tract Squamous Epithelium
}

\author{
Lewis Roy Eversole
}

Received: 16 December 2008/ Accepted: 21 January 2009/Published online: 22 February 2009

(c) Humana 2009

\begin{abstract}
Dysplasia of the oral, laryngeal and oropharyngeal stratified squamous epithelia is a microscopically defined change that may occur in clinically identifiable lesions including erythroplakia, leukoplakia and erythroleukoplakia, lesions that convey a heightened risk for carcinomatous progression. Dysplastic lesions have been classified microscopically according to degree of cytologic atypia and changes in architectural patterns, usually on a three part or four part gradation scale. Vocal cord epithelial lesions are graded according to either the Ljubljana or the World Health Organization (WHO) system whereas oral dysplasias are generally classified according to WHO criteria. Cytologically atypical cells are considered to represent precancerous changes predicting an increase risk for carcinomatous transformation. Inter- and intra-rater reliability studies among pathologists have disclosed low correlation coefficients for four part grading systems, whereas improved agreement is achieved (kappa correlation values) using the Ljubljana systems. Evidence forwarded by some studies supports the prognostic value of progressively severe dysplastic changes for carcinomatous transformation; however, some studies indicate that the presence of a clinically defined lesion without microscopic evidence of dysplasia also connotes increased risk for carcinomatous transformation. Loss of heterozygosity (LOH) at $3 p$ and $9 p$ microsatellite domains, DNA ploidy analysis and nuclear image analyses may have predictive value as molecular and histomorphological biomarkers.
\end{abstract}

L. R. Eversole $(\bowtie)$

Oral Pathology Diagnostic Services,

4945 Mercury Street, San Diego, CA 92111, USA

e-mail: lrebge@aol.com
Keywords Dysplasia - Squamous cell carcinoma . Head and neck - Larynx · Oral mucosa · Oropharynx · Biomarkers · Precancerous lesions - DNA ploidy · Loss of heterozygosity

\section{Introduction}

Precancerous lesions of the upper aerodigestive tract keratinizing epithelium include leukoplakia, erythroplakia and mixed leukoerythroplakia (hyperplastic epithelial lesions). These clinically defined lesions have been stated to harbor an increased risk compared to normal mucosa for transformation into squamous cell carcinoma. When histopathologic evidence of cytologic and architectural atypism is present in the absence of invasion, the lesions are referred to as dysplastic and the presence of dysplasia has been considered to represent an increased risk identifier for malignant transformation over that of squamous lesions that fail to show dysplastic features $[1,2]$. This review will define the microscopic features encountered in various grading systems of upper aerodigestive tract squamous dysplasia, discuss diagnostic reliability among pathologists, tabulate data on prognostication relative to carcinomatous transformation and review potential molecular and biomarker predictors.

\section{Grading Systems}

Dysplasias have been subdivided according to the degree of architectural and cytologic atypia. These grading systems have been evaluated for their prognostic utility with the assumption that mild atypical changes will have a lower risk for transformation to invasive carcinoma than those 
Fig. 1 The WHO grading system for oral and laryngeal precancerous lesions. a. Benign hyperplasia (benign keratosis), b. mild dysplasia, c. moderate dysplasia, d. severe dysplasia, e. carcinoma in situ
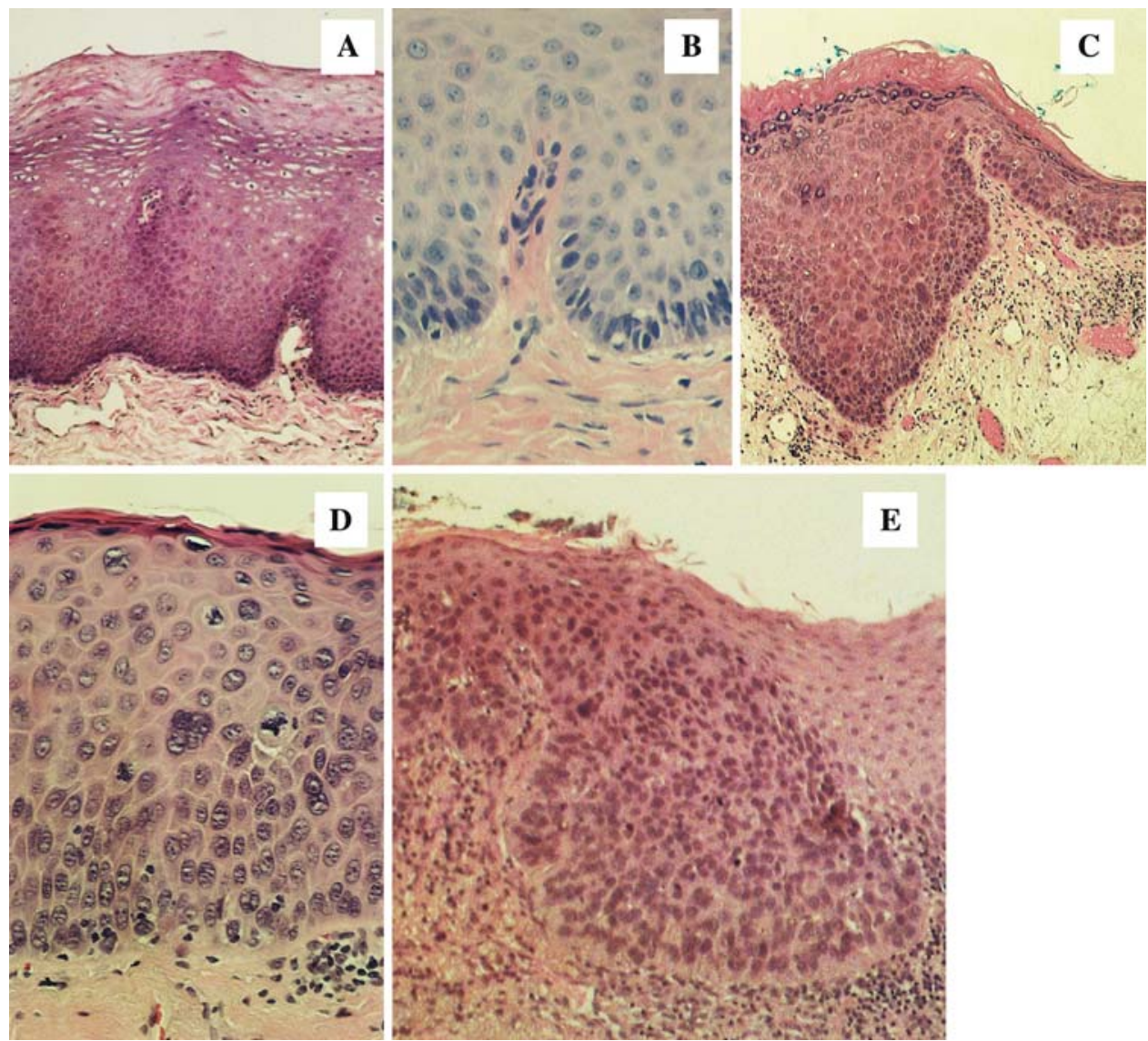

with advanced or severe changes. In the larynx, the most frequently employed systems in use among pathologists include the World Health Organization (WHO) and the Ljubljana Grading systems for vocal cord lesions; oral precancerous lesions are typically classified according to WHO [3-6]. The WHO grading system for both vocal cord and oral mucosa divides dysplasia into three categories, with the realization that dysplastic change is in reality a dynamic process. These three stages include mild, moderate and severe dysplasia flanked by hyperplasias or benign keratoses on the benign end of the spectrum and carcinoma in situ on the malignant extreme.

Low grade dysplasia is defined as cytologic and architectural atypia confined to the basal/parabasal layer; moderate dysplasia is characterized by atypical changes progressing into the mid spinous layer and severe dysplasia progresses into the upper spinous layer (Fig. 1). Carcinoma in situ is characterized by atypical changes from top to bottom. Many pathologists collapse severe dysplasia and carcinoma-in situ into a single category. Some pathologists have applied the cervical dysplasia paradigm to upper airway lesions: oral intraepithelial neoplasia (OIN 1, 2 and 3) or squamous intraepithelial neoplasia (SIN 1, 2 and 3) [7].

The Ljubljana grading system devised in Slovenia by Kambic and Gale [3,5] for laryngeal precancerous lesions divides stages of progressing dysplastic changes into three categories: "simple" hyperplasia characterized by epithelial thickening without atypical cytologic changes, "abnormal" hyperplasia represented by bulbous rete pegs and hyperplasia of the basal/parabasilar compartment with nuclear crowding and enlargement, and "atypical" hyperplasia represented by cytologic atypia extending into the upper strata (Fig. 2). Carcinoma-in situ is reserved for those lesions with pronounced top to bottom atypia and a loss of normal stratification. Table 1 compares the Ljubljana and WHO models.

\section{Grading Reliability}

If any classification scheme for upper airway precancerous lesions is to be employed or universally adopted, it should be utilitarian. This utility includes reproducibility by examiner pathologists, both between (interexaminer) and within (intraexaminer) and lesional grades should have prognostic value with regard to potential for malignant transformation to squamous cancer [7]. Investigations that assess examiner reliability are listed in Table 2. Reliability data are presented as either \% agreement or are expressed as Kappa statistics. In these studies, a group of examiners is 
Fig. 2 The Ljubljana grading system for vocal cord precancerous lesions. a. Simple hyperplasia, b abnormal hyperplasia, c. atypical (risky) hyperplasia, d carcinoma in situ
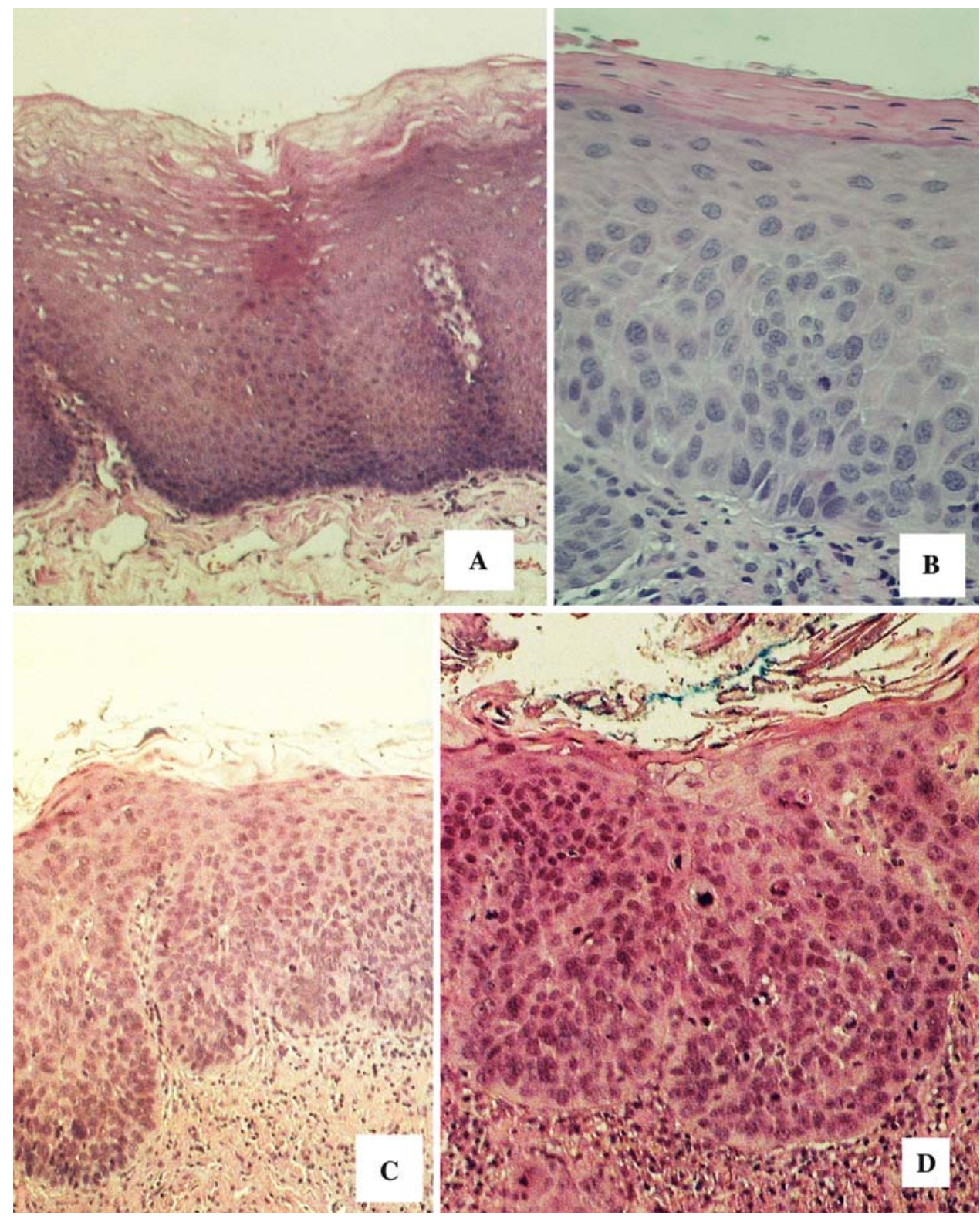

Table 1 Vocal cord dysplasia

\begin{tabular}{ll}
\hline Ljubljana System & Who System \\
\hline Simple hyperplasia & Benign keratoses \\
Abnormal hyperplasia & Mild dysplasia \\
Atypical hyperplasia & Moderate dysplasia \\
& Several dysplasia \\
\hline
\end{tabular}

asked to utilize a specified grading system and render a histopathologic diagnosis on a selection of lesions. Reponses are then compared to assess for agreement or disagreement. In those studies that address intra-rater correlations, pathologists are assigned a set of glass slides and render an initial diagnosis and subsequently, at a given future date, reevaluate the same group of slides and render a diagnosis while remaining blinded from their initial diagnoses.

In general, regardless of the grading system chosen, agreement among pathologists is only fair, kappa values falling under 0.5. Vocal cord lesional assessment employing the WHO criteria showed a poor level of agreement; however, when a binary grading system that included only two diagnostic categories (mild dysplasia versus severe dysplasia) were applied, it revealed an improvement in interrater agreement with a kappa of 0.7 [8]. Among oral precancerous lesions, using the WHO criteria, kappa values hover between 0.5 and 0.6 [7-13]. In the investigation by Kujan et al. [9] a binary grading system did not produce a higher level of agreement over the WHO system; however, in the study by Abbey et al. [11] an 80\% agreement level 
Table 2 Inter- and intra-rater reliability for dysplasia grades in oral and laryngeal precancerous lesions

\begin{tabular}{|c|c|c|c|}
\hline Authors & Grading system & $\begin{array}{l}\% \text { agreement or Kappa } \\
\text { inter-rater }\end{array}$ & $\begin{array}{l}\% \text { agreement or Kappa } \\
\text { intra-rater }\end{array}$ \\
\hline McLaren et al. (2000) [8] & VC 5 grade WHO 2 grades mild versus severe dysplasia & $\begin{array}{ll}\text { K } & 0.32 \\
\text { K } & 0.52 \\
\text { K } & 0.52\end{array}$ & \\
\hline Kujan et al. (2006) [9] & OC 2 grades 5 grade $\mathrm{WHO}$ & $\begin{array}{l}\text { K } 0.50 \\
\text { Ks } 0.22, \mathrm{Kw} 0.63\end{array}$ & \\
\hline Karabulut et al. (1995) [10] & OC 5 grade $\mathrm{WHO}$ & K $0.27-0.45(49-59 \%)$ & \\
\hline Abbey et al. (1995) [11] & OC 4 grade WHO dysplasia versus none & $\begin{array}{l}50.5 \% \\
81 \%\end{array}$ & $\begin{array}{l}50.8 \% \\
80.3 \%\end{array}$ \\
\hline Fischer et al. 2004 [12] & OC 5 grade WHO attending pathologist versus committee & K 0.59 & \\
\hline Brothwell et al. 2003 [13] & OC 5 grade WHO dysplasia versus none modal score standard & $\begin{array}{l}\text { Kw } 0.74 \\
\text { K } 0.51\end{array}$ & Ks $0.22-0.78$ \\
\hline
\end{tabular}

$\mathrm{VC}=$ vocal cords, $\mathrm{OC}=$ oral cavity; 2 grades—low grade dysplasia/high grade dysplasia; Ljubljana—simple hyperplasia, abnormal hyperplasia, atypical hyperplasia, carcinoma in situ; 5 grade WHO — no, mild, moderate, severe dysplasias and carcinoma in situ; 4 grade merges severe dysplasia with CIS

was realized when pathologists were asked to evaluate cases that were dysplastic (any grade) compared to cases without dysplasia. A similar level of agreement was observed within pathologists (intra-rater).

These data are somewhat disappointing, being nonreproducible in many instances. If pathologists cannot agree on diagnoses for which clinicians plan treatment strategies, then the system somehow fails unless all lesions regardless of histology are treated in the same fashion. It is noteworthy that certain variables factor into disagreement among pathologists, namely presence or absence of inflammation, lesion site and biopsy method [12]. When pathologists undergo a group training session on what histopathologic, cytologic and architectural features are used as criteria for each grade of dysplasia/hyperplasia, subsequent agreement reliability significantly improves [14].

\section{Prediction of Malignant Transformation}

Prognostication should be the outcome goal of histopathologic grading of precancerous lesions of the upper air passages. Many studies have been undertaken to determine the prevalence of carcinomatous transformation in clinically defined leukoplakia, erythroplakia, acute laryngitis (vocal cord erythroplakia) and chronic laryngitis (vocal cord leukoplakia) [14-23] (Table 3). Five recent follow-up studies on vocal cord lesions report a range of 3.8-11.2\% malignant transformation rate for all lesions regardless of histopathology (i.e. both dysplastic and nondysplastic hyperplasias); a 10.5-32\% rate for lesions with histologic evidence of dysplasia has been reported [14-18]. When grade of dysplasia using either the WHO or Ljubljana systems are assessed, there is a progressive increase in carcinomatous transformation rate with increasing severity of dysplastic change. Data on oral mucosal dysplasias, based on five follow-up studies discloses a malignant transformation rate range for combined dysplastic and nondysplastic lesions of 3.9-17\% [1, 19-22]. In the study by Silverman et al. [1], the risk for cancer progression in leukoplakias jumped from $17 \%$ for all lesions to $36 \%$ for dysplastic lesions whereas the remaining studies failed to find any statistically significant increased risk for dysplastic lesions [19-22]. Holmstrup et al. [20] demonstrated a progressive increase in risk correlated with increasing degrees of dysplasia; however, their population sample was too low to achieve statistical significance. Lastly, it is noteworthy that the vast majority of precancerous lesions arising on vocal cord and oral mucosae, regardless of whether dysplastic changes are extant, do not progress to carcinoma, at least within the follow-up period specified in various reports.

\section{Molecular Biomarkers and Prediction}

A variety of proliferation markers, cell cycle cyclin proteins, cyclin kinases, oncoproteins, tumor suppressor mutations, microsatellite loss of heterozygosity ( $\mathrm{LOH}$ ), nuclear image parameters and DNA ploidy have been investigated in both oral and laryngeal carcinomas as well as dysplasias [17, 23, 24]. These investigations have provided insight into the molecular mechanisms of carcinogenesis. It is beyond the scope of this communication to review this literature, and therefore only those with promise as potential biomarker predictors will be discussed. It is noteworthy that immunohistochemical 
Table 3 Progression to carcinoma from dysplastic precancerous lesions of the laryngeal and oral mucosa (percentage)

\begin{tabular}{|c|c|c|c|c|c|c|c|c|c|}
\hline \multirow[t]{2}{*}{ Author } & \multirow[t]{2}{*}{ Location } & \multirow[t]{2}{*}{ All lesions dysp/nondysp } & \multirow[t]{2}{*}{ All dysplasia } & \multicolumn{5}{|c|}{ Grade of dysplasia $^{a}$} & \multirow[t]{2}{*}{ FU (years) } \\
\hline & & & & 0 & 1 & 2 & 3 & 4 & \\
\hline \multirow[t]{2}{*}{ Hellquist et al. (1999) [14] } & Larynx & & & & & & & & \\
\hline & $n=899$ & 11.2 & 10.5 & 0.7 & - & 1 & 9.5 & - & $1.5-12$ \\
\hline \multirow[t]{3}{*}{ Isenberg et al. (2008) [15] } & Larynx & & - & - & - & - & - & - & - \\
\hline & $n=208$ & 3.8 & 33 & - & - & - & - & - & - \\
\hline & $n=2188^{\mathrm{b}}$ & 3.7 & & 4 & & 10 & 18 & & \\
\hline \multirow[t]{2}{*}{ Ricci et al. (2003) [16] } & Larynx & & & & & & & & \\
\hline & $n=207$ & - & 17 & - & - & - & - & - & $7-16$ \\
\hline \multirow[t]{2}{*}{ Jeannon et al. (2004) [17] } & Larynx & & 24 & & & & & & \\
\hline & $n=114$ & & & - & - & - & - & - & - \\
\hline Gale et al. (2000) [18] & Larynx & & & 0.9 & - & - & - & 11 & \\
\hline Hsue et al. (2007) [19] & $\begin{array}{l}\text { Oral } \\
n=1,458\end{array}$ & 3.9 & 4.8 & 3 & - & - & - & - & 3.5 \\
\hline Holmstrup et al. (2006) [20] & $\begin{array}{l}\text { Oral } \\
n=101\end{array}$ & 12 & 12 & 4 & 4 & 8 & 17 & 20 & 6.8 \\
\hline Silverman et al. (1984) [1] & $\begin{array}{l}\text { Oral } \\
n=257\end{array}$ & 17 & 36 & & & & & & 7.2 \\
\hline \multirow[t]{2}{*}{ Schepman et al. (1998) [21] } & Oral & & & & & & & & \\
\hline & $n=166$ & 2.9 annual & & & & & & & 2.4 \\
\hline \multirow[t]{2}{*}{ Hogewind et al. (1989) [22] } & Oral & & & & & & & & \\
\hline & $n=46$ & 6.5 & 2.2 & & & & & & 5 \\
\hline
\end{tabular}

${ }^{a} 0=$ benign keratosis; $1=$ mild dysplasia; $2=$ moderate dysplasia; $3=$ severe dysplasia; $4=$ carcinoma in situ. Ljubljana $0=$ simple hyperplasia; 1 = abnormal hyperplasia; $2,3=$ atypical hyperplasia; 4 = carcinoma in situ

b Original cases with literature review

markers that mirror the changes observed microscopically are of no prognostic value. Only a marker that can be identified in premalignant lesions whose presence predicts progression offers any meaningful clinical application. Three approaches have shown utility for upper airway prognostication including the presence of aneuploidy, microsatellite instability with $\mathrm{LOH}$ at $3 p$ and $9 p$ and computer generated nuclear image analysis [25-27].

Crissman and Zarbo [25] demonstrated a direct correlation between the presence of aneuploidy and degree of dysplasia in vocal cord lesions, with all severely dysplastic lesions being aneuploid. Unfortunately, this study was not progressive and did not address predictive follow-up information. No reliable ploidy data have been forthcoming in any large follow-up studies of oral mucosal dysplastic lesions. Rosin et al. [26] investigated hyperplastic and mild dysplastic oral lesions, assessing lesions that progressed to carcinoma and those that failed to progress using microsatellite LOH as a biomarker. They reported a 33 fold elevated cancer risk for lesions that progressed to cancer when LOH at $3 p$ and/or 9p coupled with additional losses at $4 \mathrm{q}, 8 \mathrm{p}, 11 \mathrm{q}$, or $17 \mathrm{p}$ were identified. Guillaud et al. demonstrated that this molecular predictive feature could be further augmented when combining $\mathrm{LOH}$ data with nuclear image analysis (nuclear phenotype score).

\section{Discussion}

Based upon current knowledge, the majority of dysplastic oral and vocal cord lesions do not progress to squamous cancer, at least in a mean follow-up period of 7 years and therefore the terms "intraepithelial neoplasia", either oral or squamous are to be discouraged for head and neck lesions. Even the issue of an increase in carcinomatous transformation prevalence among dysplastic as compared to benign hyperplastic lesions may be questionable for oral precancerous lesions. Alternatively, an increased cancer risk in vocal cord dysplasias is extant over nondysplastic laryngeal epithelial hyperplasias, although not many long term follow-up studies have been published.

Some studies do report an increased risk for cancer when dysplasia is encountered in oral mucosal leukoplakias and erythroplakias, although only one study compared risk according to grade of dysplasia, failing to find a statistically significant correlation. Prognosis and prediction 
for cancer progression from precancerous lesions will continue to be based on features of dysplasia until more definitive markers can be discovered. Carcinoma-in situ should be considered a true "intraepithelial" neoplastic process and further investigations are needed to assess this severest variant of dysplastic change as a probable predictor.

Biomarkers with potential predictive value have been published and may prove to be clinically relevant. These include DNA ploidy, computerized nuclear image analysis and micro satellite instability at $3 p$ and $9 p$ with addition $\mathrm{LOH}$ at other loci.

\section{References}

1. Silverman S Jr, Gorsky M, Lozada F. Oral leukoplakia and malignant transformation. A follow-up study of 257 patients. Cancer. 1984;53:563-8.

2. Hellquist H, Lundgren J, Olofsson J. Hyperplasia, keratosis, dysplasia and carcinoma in situ of the vocal cords: a follow-up study. Clin Otolaryngol. 1982;7:11-27.

3. Sengiz S, Pabuccuoglu U, Sulen S. Immunohistological comparison of the World Health Organization (WHO) and Ljubljana classifications on the grading of preneoplastic lesions of the larynx. Pathol Res Pract. 2004;200:181-8.

4. Shanmugaratnam K in collaboration with Sabin LH et al. Histologic typing of tumors of the upper respiratory tract and ear. Berlin: Springer Verlag; 1991.

5. Michaels L. The Kambic-Gale method of assessment of epithelial hyperplastic lesions of the larynx in comparison with the dysplasia grade method. Acta Otolaryngol. 1997;527:17-20.

6. World Health Organization Classification of Tumours. Pathology \& genetics. Head and neck tumors. International agency for research in cancer (IARC). In: Barnes L, Eveson JW, Reichart P, Sidransky D, editors. Head and Neck Tumors. Lyon: IARC Press; 2005 p. $177-80$.

7. Warnakulasuriya S, Reibel J, Bouquot J, Dabelsteen E. Oral epithelial dysplasia classification systems: predictive value, utility, weaknesses and scope for improvement. J Oral Pathol Med. 2008;17:127-33.

8. McLaren KM, Burnett RA, Goodlad JR, Howatson SR, Lang S, Lee FD, et al. Consistency of histopathological reporting of laryngeal dysplasia. Histopathology. 2000;17:460-3.

9. Kujan O, Oliver RJ, Khattab A, Roberts SA, Thakker N, Sloan P. Evaluation of a new binary system of grading oral epithelial dysplasia for prediction of malignant transformation. Oral Oncol. 2006;42:987-93.

10. Karabulut A, Reibel J, Therkildsen MH, Praetorius F, Nielsen HW, Dabelsteen E. Observer variability in the histologic assessment of oral premalignant lesions. J Oral Pathol Med. 1995;24:198-200.

11. Abbey LM, Kaugars GE, Gunsolly JC, et al. Intraexaminer and interexaminer reliability in the diagnosis of oral epithelial dysplasia. Oral Surg Oral Med Oral Pathol Oral Radiol Endod. 1995;80:188-91.

12. Fischer DJ, Epstein JB, Morton TH, Schwartz SM. Interobserver variability in the histopathologic diagnosis of oral pre-malignant and malignant lesions. J Oral Pathol Med. 2004;33:65-70.

13. Brothwell DJ, Lewis DW, Bradley G, Leong I, Jordan RC, Mock $\mathrm{D}$, et al. Observer agreement in the grading of oral epithelial dysplasia. Community Dent Oral Epidemiol. 2003;31:300-5.

14. Hellquist H, Cardesa A, Gale N, Kambic V, Michaels L. Criteria for grading in the Ljubljana classification of epithelial hyperplastic laryngeal lesions. A study by members of the working group on epithelial hyperplastic laryngeal lesions of the European Society of Pathology. Histopathology. 1999;34:226-33.

15. Isenberg JS, Crozier DL, Dailey SH. Institutional and comprehensive review of laryngeal leukoplakia. Ann Otol Rhinol Laryngol. 2008;117:74-9.

16. Ricci G, Molini E, Faralli M, Simoncelli C. Retrospective study on precancerous laryngeal lesions: long-term follow-up. Acta Otorhinolaryngol Ital. 2003;23:362-7.

17. Jeannon JP, Soames JV, Aston V, Stafford FW, Wilson JA. Molecular markers in dysplasia of the larynx: expression of cyclin-dependent kinase inhibitors p21, p27 and p53 tumour suppressor gene in predicting cancer risk. Clin Otolaryngol Allied Sci. 2004;29:698-704.

18. Gale N, Kambic V, Michaels L, Cardesa A, Hellquist H, Zidar N, et al. The Ljubljana classification: a practical strategy for the diagnosis of laryngeal precancerous lesions. Adv Anat Pathol. 2000;7:240-51.

19. Hsue S-S, Wang W-C, Chen C-H, Lin C-C, Chen Y-K, Lin L-M. Malignant transformation in 1458 patients with potentially malignant oral mucosal disorders: a follow-up study based in a Taiwanese hospital. J Oral Pathol Med. 2007;36:25-9.

20. Holmstrup P, Vedtofte P, Reibel J, Stoltze K. Long-term treatment outcome of oral premalignant lesions. Oral Oncol. 2006;42:461-74.

21. Schepman KP, van der Meij EH, Smeele LE, van der Waal I. Malignant transformation of oral leukoplakia: a follow-up study of a hospital-based population of 166 patients with oral leukoplakia in the Netherlands. Oral Oncol. 1998;34:270-5.

22. Hogewind WF, van der Kwast WA, van der Waal I. Oral leukoplakia with emphasis on malignant transformation. A followup study of 46 patients. J Craniomaxillofac Surg. 1989;17:12833.

23. Ioachim E, Peschos D, Goussia A, Mittari E, Charalabopoulos K, Michael M, et al. Expression patterns of cyclins D1, E in laryngeal epithelial lesions: correlation with other cell cycle regulators (p53, pRb, Ki-67 and PCNA) and clinicopathological features. J Exp Clin Cancer Res. 2004;23:277-83.

24. Forastiere A, Koch W, Trotti A, Sidransky D. Head and neck cancer. N Engl J Med. 2002;346:1416-7.

25. Crissman JD, Zarbo RJ. Quantitation of DNA ploidy in squamous intraepithelial neoplasia of the laryngeal glottis. Arch Otolarygol Head Neck Surg. 1991;117:182-8.

26. Rosin MP, Cheng X, Poh C, Lam WL, Huang Y, Lovas J, et al. Use of allelic loss to predict malignant risk for low-grade oral epithelial dysplasia. Clin Cancer Res. 2000;6:357-62.

27. Guillaud M, Shang L, Poh C, Rosin MP, MacAulay C. Potential use of quantitative tissue phenotype to predict malignant risk for oral premalignant lesions. Cancer Res. 2008;68:3099-107. 